\title{
Predictors of aortic stenosis severity reclassification using an imaging data fusion method in patients referred for transcatheter aortic valve implantation
}

\author{
Patrycjusz Stokłosa ${ }^{1}$, Ilona Michałowska², Piotr Duchnowski ${ }^{1}$, Małgorzata Ryś ${ }^{1}$, \\ Agnieszka Żebrowska1 ${ }^{1}$ Ewa Orłowska-Baranowska ${ }^{1}$, Tomasz Hryniewiecki ${ }^{1}$, Piotr Szymański ${ }^{1}$ \\ 'Department of Valvular Heart Disease, The Cardinal Stefan Wyszynski Institute of Cardiology, Warsaw, Poland \\ ${ }^{2}$ Department of Radiology, The Cardinal Stefan Wyszynski Institute of Cardiology, Warsaw, Poland
}

\begin{abstract}
Background: The use of imaging data fusion method (IDFM) with multislice computed tomography (MSCT) and two-dimensional transthoracic echocardiography (2D-TTE) in patients with aortic stenosis (AS) may result in reclassification of AS severity from severe to non-severe.
\end{abstract}

Aim: We sought to establish potential predictors of AS severity reclassification using the IDFM method.

Methods: A total of 54 high-risk patients (mean age $79 \pm 7.9$ years; 40.7\% male) with severe AS by 2D-TTE (indexed aortic valve area $[A V A i]<0.6 \mathrm{~cm}^{2} / \mathrm{m}^{2}$ ), referred for transcatheter aortic valve implantation, were included in the analysis. AVAi was subsequently recalculated using IDFM by replacing 2D-TTE left ventricular outflow tract (LVOT) measurements with MSCT LVOT parameters.

Results: Imaging data fusion method reclassified $20.4 \%$ patients into the potentially non-severe AS group. In a multivariable model including clinical variables, reclassification to non-severe AS by IDFM was independently associated with younger age and diabetes mellitus (DM), (odds ratio [OR] 0.864; 95\% confidence interval [CI] 0.76-0.99; $\mathrm{p}<0.035$ and OR 19.259; 95\% Cl 2.28-162.41; $\mathrm{p}<0.007$, respectively). In a multivariable analysis of echocardiographic variables, reclassification was associated with higher LVOT velocity time integral and lower aortic mean gradient (OR 1.402; 95\% Cl 1.07-1.84; $\mathrm{p}<0.014$ and OR 0.858 ; 95\%: Cl 0.760-0.968; $\mathrm{p}<0.013$, respectively). In addition, $24.1 \%$ of patients were reallocated from low-flow $\left(<35 \mathrm{~mL} / \mathrm{m}^{2}\right)$ to normal-flow AS.

Conclusions: Imaging data fusion method reclassified a substantial proportion of patients with severe AS into a potentially moderate AS group and from a low-flow to a normal-flow AS group. Such regrouping calls for increased diagnostic prudence in AS patients, especially those with specific clinical and echocardiographic predictors of reclassification, such as DM or low aortic mean gradient.

Key words: aortic valve stenosis, echocardiography, multimodality imaging, multislice computed tomography, reclassification, transcatheter aortic valve implantation

Kardiol Pol 2018; 76, 12: 1725-1732

\section{INTRODUCTION}

For more than a decade transcatheter aortic valve implantation (TAVI) has been a rescue procedure for patients with severe symptomatic aortic stenosis (AS) not suitable for surgical aortic valve replacement. The results are encouraging [1, 2].
Multimodal imaging of the aortic valve complex in this group of patients is a necessity [3, 4]. However, two-dimensional transthoracic echocardiography (2D-TTE), two-dimensional transoesophageal echocardiography (2D-TEE), three-dimensional TEE (3D-TEE), and multislice computed tomography 

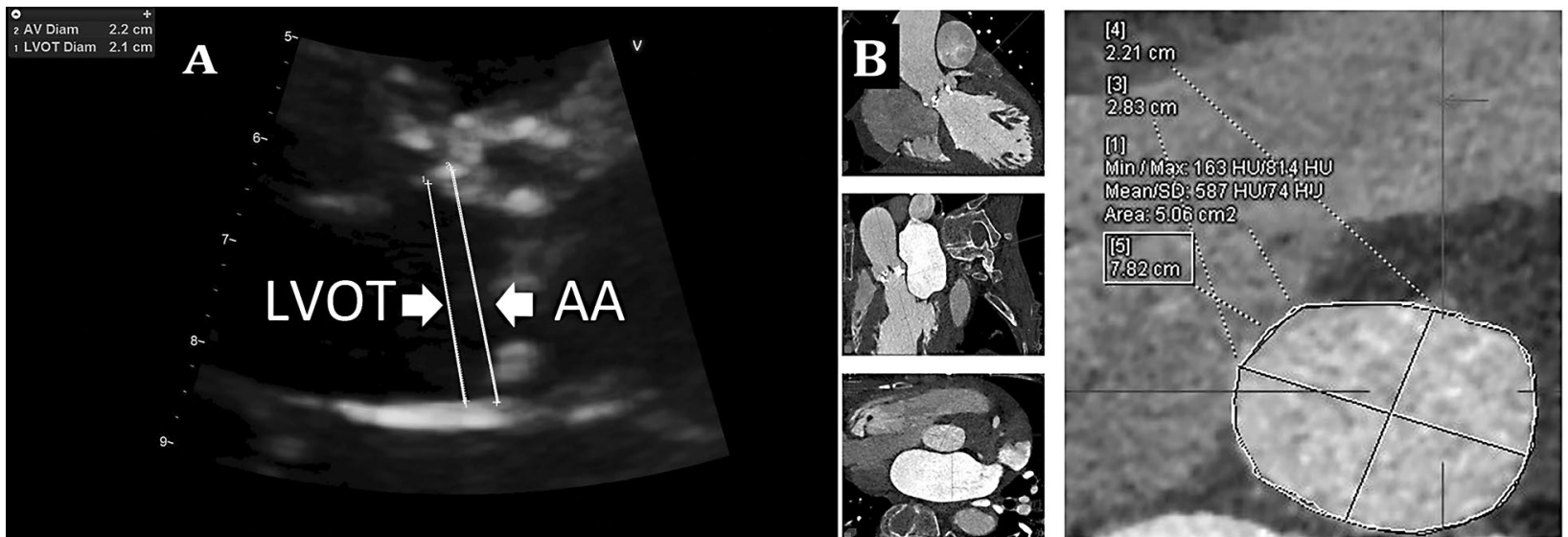

Figure 1. Left ventricular outflow tract (LVOT) and aortic annulus (AA) assessed using two-dimensional transthoracic echocardiography (2D-TTE) (A) and multislice computed tomography (MSCT) (B). In 2D-TTE AA was measured at hinge-points (trailing edge to leading edge convention) and distal LVOT (inner edge to inner edge convention) as the widest diameter just beneath the AA plane. In MSCT AA was manually traced in the plane at the lowest portions of the aortic leaflets and distal LVOT, $5 \mathrm{~mm}$ beneath the nadirs of the cusps (both in the blood-tissue interface convention)

(MSCT) imaging differ significantly with regard to the precision of left ventricular outflow tract (LVOT) [5-7] and aortic annulus (AA) $[3,8,9]$ measurements, with MSCT considered to be the most exact imaging tool $[3,4,10]$. Using the most accurate MSCT LVOT size in an imaging data fusion method (IDFM) - combining the MSCT LVOT area and 2D-TTE Doppler parameters - leads to a recalculation of stroke volume (SV) and aortic valve area (AVA), as shown by Kamperidis et al. [8] and Clavel et al. [7]. As a result, some patients are reclassified as having non-severe rather than severe AS. While potentially of significant clinical importance, clinical and anatomical predictors of such reclassification have not been determined yet.

\section{METHODS}

\section{Patients}

A total of 54 high-risk patients with symptomatic severe AS (indexed AVA [AVAi] $<0.6 \mathrm{~cm}^{2} / \mathrm{m}^{2}$ ) diagnosed by $2 \mathrm{D}-\mathrm{TTE}$ referred by the local heart team for the TAVI procedure (2014-2015) were included in the study. All patients underwent 2D-TTE and MSCT examinations within a median of 22 days with no or minor pharmacotherapy interventions that should not impact the examination outcomes or IDFM calculations. Informed consent for the diagnostic and treatment procedures needed for TAVI was obtained from all patients. The study was approved by the local Research Ethics Committee.

\section{Echocardiography}

Echocardiography was carried out according to current guidelines [11-13]. The examinations were performed with commercially available ultrasound systems (Vivid S70 and E9; General Electric Medical Systems, Milwaukee, WI, USA). Measurements were done with a dedicated workstation
(EchoPAC version 201; General Electric Medical Systems). In 2D-TTE left ventricular (LV) measurements were performed in a 2D-mode and LV ejection fraction was assessed with the biplane method of disks (modified Simpson's rule). LVOT and AA diameters were measured in long-axis, in zoom mode, in mid-systole, in three consecutive cardiac cycles. AA was measured first - at hinge-points and distal LVOT as the widest diameter just beneath the AA plane - about 3 to $5 \mathrm{~mm}$ from the AA posterior hinge-point and $1-2 \mathrm{~mm}$ from the anterior AA hinge-point (Fig. 1A).

Stroke volume, SV indexed to body surface area (SVi), AVA, and AVAi were calculated with the continuity equation. High-gradient AS was diagnosed when aortic mean gradient (AMG) was higher than $40 \mathrm{mmHg}$. Patients were classified into the low-flow group when calculated SVi, based on 2D-TTE Doppler, was $<35 \mathrm{~mL} / \mathrm{m}^{2}$. 2D-TEE with 3D-TEE imaging was used for confirmation of the diagnosis in low-gradient groups and for comparison purposes in high-gradient patients.

\section{Multislice computed tomography}

Multislice computed tomography examinations were performed with the use of the Somatom Force device (Siemens Medical Solutions, Erlangen, Germany; number of slices: $2 \times 192$; rotation time: $250 \mathrm{~ms}$; $\mathrm{kV}$ steps: 70-150 kV; slice thickness: $0.6 \mathrm{~mm}$ ). Technical parameters were adjusted to body weight. An injection of 45-70 mL of nonionic iodinated contrast agent (with $370-400 \mathrm{mgl} / \mathrm{mL}$ ) at $4-5 \mathrm{~mL} / \mathrm{s}$ into the antecubital vein was administered, followed by a $30-\mathrm{mL}$ saline bolus. Scan data were then reconstructed using retrospective electrographic gating from mid-systole (35\%-45\% R-R interval). Measurements were done with a dedicated Siemens workstation, syngo.via. To measure the $\mathrm{AA}$, the double-oblique transverse plain was realigned to be perpendicular to the long axis of the aortic 
Table 1. Baseline clinical characteristics of the study subjects $(n=54)$

\begin{tabular}{|lc|} 
Variable & Value \\
\hline Age [years] & $79.9 \pm 7.9$ \\
Male sex & $22(40.7)$ \\
Arterial hypertension & $44(81.5)$ \\
BMI [kg/m²] & $27.7 \pm 5.2$ \\
BSA [m²] & $1.9 \pm 0.2$ \\
CAD & $35(64.8)$ \\
Previous MI & $17(31.5)$ \\
Previous CABG & $12(22.2)$ \\
COPD & $8(14.8)$ \\
Diabetes mellitus & $20(37.1)$ \\
Creatinine level $[\mu \mathrm{mol} / \mathrm{L}]$ & $101.6 \pm 49.8$ \\
eGFR [mL/min/1.73 $\left.\mathrm{m}^{2}\right]$ & $58.3 \pm 2.5$ \\
Haemoglobin level $[\mathrm{g} / \mathrm{dL}]$ & $12.5 \pm 1.4$ \\
NYHA class II & $23(42.6)$ \\
NYHA class III & $26(48.1)$ \\
NYHA class IV & $5(9.3)$ \\
EuroSCORE II [\%] & $18.8 \pm 12.8$ \\
\hline
\end{tabular}

Values are presented as mean \pm standard deviation or number (percentages). BMI — body mass index; BSA — body surface area; CABG — coronary artery bypass grafting; $C A D$ — coronary artery disease; COPD — chronic obstructive pulmonary disease; eGFR — estimated glomerular filtration rate; EuroSCORE — European System for Cardiac Operative Risk Evaluation; MI — myocardial infarction; NYHA — New York Heart Association

root at the lowest portions of the aortic leaflets. At this level, AA boundaries were manually traced (Fig. 1B). For distal LVOT measurements the transverse plane was set lower, so as to be $5 \mathrm{~mm}$ beneath the nadirs of the cusps. MSCT AA and LVOT areas were planimetered and calculated from coronal (maximal) and sagittal (minimal) diameters using the formula for the area of a circle ( $r$ was calculated as $1 / 2$ of the mean diameter).

\section{Imaging data fusion method}

In IDFM, fusion SVi (SVFi) and fusion AVAi (AVAFi) were calculated with a modified continuity equation using Doppler LVOT velocity time integral $\left(\mathrm{VTI}_{\mathrm{LVOT}}\right)$ derived from 2D-TTE and maximum LVOT cross-sectional area derived from MSCT measurements.

\section{Reclassification}

Patients were reclassified to the non-severe AS group when AVAi calculated based on IDFM was $\geq 0.6 \mathrm{~cm}^{2} / \mathrm{m}^{2}$, and to the normal-flow group when SVFi established using IDFM was $\geq 35 \mathrm{~mL} / \mathrm{m}^{2}$.

\section{Statistical analysis}

Statistical analysis was performed with the IBM SPSS software, version 2.0 (SPSS Inc., Chicago, IL, USA). A p-value
Table 2. Baseline echocardiographic data -2 D-TTE $(n=54)$

\begin{tabular}{|c|c|}
\hline Variable & Value \\
\hline APG $[\mathrm{mmHg}]$ & $86.1 \pm 23.8(31-166)$ \\
\hline AMG $[\mathrm{mmHg}]$ & $52.7 \pm 16.3(19-115)$ \\
\hline$V_{T I}{ }_{\text {LVOT }}$ & $22.6 \pm 5.4(11.4-37.0)$ \\
\hline $\mathrm{VTI}_{\mathrm{AV}}$ & $114.1 \pm 23.3(56.3-190.9)$ \\
\hline $\mathrm{SV}_{2 \mathrm{D}-\mathrm{TTE}}[\mathrm{mL}]$ & $74.2 \pm 27.3(32.4-218.3)$ \\
\hline$S V i_{2 D-T T E}\left[\mathrm{~mL} / \mathrm{m}^{2}\right]$ & $39.8 \pm 12.7(18.3-100.5)$ \\
\hline $\mathrm{AVA}_{2 \mathrm{D}-\mathrm{TTE}}\left[\mathrm{cm}^{2}\right]$ & $0.66 \pm 0.24(0.33-2.01)$ \\
\hline $\mathrm{AVAi}_{2 \mathrm{D} \text {-TTE }}\left[\mathrm{cm}^{2} / \mathrm{m}^{2}\right]$ & $0.36 \pm 0.11(0.20-0.93)$ \\
\hline $\mathrm{AA}[\mathrm{mm}]$ & $22.9 \pm 2.0(19-31)$ \\
\hline LVOT [mm] & $20.3 \pm 2.4(15-30)$ \\
\hline LVEF [\%] & $59.2 \pm 11.4(30-75)$ \\
\hline PASP $[\mathrm{mmHg}]$ & $48.6 \pm 15.5(23-99)$ \\
\hline Moderate to severe MR & $5(9.3)$ \\
\hline Moderate to severe AR & $0(0)$ \\
\hline Low-flow AS & $16(29.6)$ \\
\hline Low-gradient AS & $11(20.4)$ \\
\hline
\end{tabular}

Data are presented as mean \pm standard deviation (range) or number (percentage). 2D-TTE - two-dimensional transthoracic echocardiography; AA — aortic annulus; AMG — aortic mean pressure gradient; APG — aortic peak pressure gradient; AR - aortic regurgitation; AS aortic stenosis; $\mathrm{AVA}_{2 \mathrm{D}-\mathrm{TTE}}$ - aortic valve area in 2D-TTE; $\mathrm{AVAi}_{2 \mathrm{D}-\mathrm{TT}}$ indexed AVA in 2D-TTE; LVEF — left ventricular ejection fraction; LVOT - left ventricular outflow tract; MR - mitral regurgitation; PASP pulmonary artery systolic pressure; $\mathrm{SV}_{2 \mathrm{D}-\mathrm{TT}}$ - stroke volume in 2D-TTE; $\mathrm{SVi}_{2 \mathrm{D}-\mathrm{TTE}}$ - indexed $\mathrm{SV}$ in $2 \mathrm{D}-\mathrm{TTE} ; \mathrm{VTI}_{A V}$ - aortic valve velocity time integral; $\mathrm{VTI}_{\text {LVOT }}$ - left ventricular outflow tract velocity time integral

of $<0.05$ was considered to be statistically significant. Continuous data were expressed as mean \pm standard deviation, and categorical variables as numbers or percentages. The association between categorical variables was evaluated with the $\chi^{2}$ test or Fisher exact test, as appropriate. Continuous variables were compared by the paired Student $t$ test. Correlations between continuous variables were established with the Pearson test. Bland-Altman analysis was performed to determine the bias and the limits of agreement between two measurements. In order to find predictors of reclassification, an AVAi univariable analysis of clinical and anatomic variables was performed with binary logistic regression analysis, and variables with a p-value of less than 0.1 were introduced in the multivariable models. Individual models were built for clinical and imaging parameters (separately for 2D-TTE and MSCT), to limit the number of variables in a model.

\section{RESULTS \\ Patients}

Baseline clinical and echocardiographic characteristics of the study group are provided in Tables 1 and 2 .

Overall, 34 (63\%) patients were classified as normal-flow high-gradient (NFHG), four (7.4\%) as normal-flow low-gradient (NFLG), six (11.1\%) as low-flow low-gradient (LFLG), 
Table 3. Left ventricular outflow tract (LVOT) diameters and areas assessed by two-dimensional transthoracic echocardiography (2D-TTE) and multislice computed tomography (MSCT) $(n=54)$

\begin{tabular}{|c|c|c|c|c|}
\hline & Mean & SD & Minimum & Maximum \\
\hline \multicolumn{5}{|l|}{ LVOT diameter [mm]: } \\
\hline $\mathrm{LVOT}_{2 \mathrm{D}-\mathrm{TTE}}$ & 20.3 & 2.4 & 15.0 & 30.0 \\
\hline LVOT $_{\text {MSCTmin }}$ & 20.2 & 2.8 & 13.0 & 30.0 \\
\hline LVOT $_{\text {MSCTmax }}$ & 28.1 & 3.0 & 23.0 & 35.0 \\
\hline $\mathrm{LVOT}_{\text {MSCTmean }}$ & 24.1 & 2.7 & 18.0 & 32.0 \\
\hline \multicolumn{5}{|l|}{ LVOT area $\left[\mathrm{mm}^{2}\right]$ : } \\
\hline LVOTarea $_{\text {2D-TTE }}$ & 328.9 & 82.3 & 176.6 & 706.5 \\
\hline LVOTarea $_{\text {MScTplanimetry }}$ & 460.0 & 104.7 & 258.0 & 738.0 \\
\hline LVOTarea $_{\text {MSCTirc }}$ & 464.4 & 104.0 & 254.3 & 803.8 \\
\hline
\end{tabular}

LVOT $_{2 \mathrm{D}-\mathrm{TTE}}$ - left ventricular outflow tract diameter in two-dimensional transthoracic echocardiography; LVOT $_{\text {MSCTmin }}$ - LVOT minimal diameter in




LVOTarea $_{\text {MSCTirc }}$ - LVOT area in MSCT calculated with the $\pi \mathrm{r}^{2}$ formula for minimal and maximal diameter; SD — standard deviation

and $10(18.5 \%)$ as low-flow high-gradient (LFHG). Therefore, in total the low-flow group consisted of 16 (29.6\%) patients.

\section{Comparison of 2D-TTE and MSCT}

Left ventricular outflow tract measurements performed with 2D-TTE and MSCT are presented in Table 3. MSCT showed the largest LVOT areas. There were moderate correlations between the MSCT LVOT mean diameter and the 2D-TTE LVOT diameter $(r=0.65, p<0.001)$ and moderately strong correlations between MSCT LVOT area and 2D-TTE LVOT area $(r=0.68, p<0.001)$. There were strong correlations between SVFi and 2D-TTE SVi $(r=0.79, p<0.001$; Fig. 2A) as well as AVAFi and 2D-TTE AVAi ( $r=0.76, p<0.001$; Fig. 2B).

\section{Discordance between 2D-TTE and}

IDFM in SVi and AVAi measurements

The differences in LVOT diameters and areas as measured with the two imaging methods translated to differences in AVAi $\left(0.36 \pm 0.11 \mathrm{~cm} / \mathrm{m}^{2}\right.$ vs. $\left.0.50 \pm 0.13 \mathrm{~cm} / \mathrm{m}^{2}\right)$ and SVi $\left(39.8 \pm 12.7 \mathrm{~mL} / \mathrm{m}^{2}\right.$ vs. $\left.51.1 \pm 13.2 \mathrm{~mL} / \mathrm{m}^{2}\right)$ calculations between 2D-TTE and IDFM, with both AVAi and SVi being systematically higher when assessed with IDFM (Fig. 2C, D).

\section{Correlations between AVAi and AMG for 2D-TTE and IDFM}

A statistically significant correlation between AMG and AVAi was observed for IDFM but not for 2D-TTE (Fig. 3).

\section{Reclassification of aortic stenosis severity and flow status}

By using AVAFi of $\geq 0.6 \mathrm{~cm}^{2} / \mathrm{m}^{2}$ as a cut-off value, $20.4 \%$ of patients with severe AS in 2D-TTE were reclassified into the non-severe AS group (Fig. 4).
In univariable models, including clinical and imaging data, reclassification was independently associated with younger age, male sex, coronary artery disease (CAD) with previous myocardial infarction $(\mathrm{MI})$, previous coronary artery bypass grafting (CABG), diabetes mellitus (DM), and lower aortic valve $\mathrm{VTI}\left(\mathrm{VTI}_{\mathrm{AV}}\right)$ and $\mathrm{AMG}$ values, but with higher values of $\mathrm{VTI}_{\mathrm{LVOT}^{\prime}}, \mathrm{MSCT}$ planimetered AA area (AAarea MSCTplanimetry ), minimal MSCT LVOT diameter (LVOT $_{\text {MSCTmin }}$ ), mean MSCT

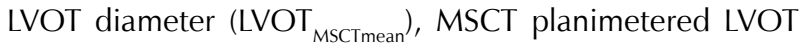

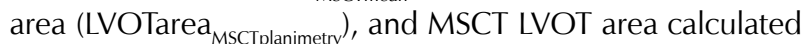
with the $\pi r^{2}$ formula (LVOTarea ${ }_{\text {MSCTcirc }}$ ) (Table 4).

In a multivariable model including clinical variables, AS reclassification was independently associated with age (odds ratio [OR] 0.864, 95\% confidence interval [Cl] 0.76-0.99; $\mathrm{p}<0.035)$ and DM (OR 19.259, 95\% Cl 2.28-162.41; $p<0.007)$.

In a multivariable model including echocardiographic variables, independent predictors of AS reclassification were $\mathrm{VTI}_{\text {LVOT }}(\mathrm{OR} 1.402,95 \% \mathrm{Cl} 1.07-1.84 ; \mathrm{p}<0.014)$ and AMG (OR 0.858, 95\%: Cl 0.760-0.968; $p<0.013$ ).

In a multivariable model including MSCT data, LVOTMSCTmin was identified as an independent predictors of AS reclassification (OR 3.901, 95\% Cl 1.08-14.16; $p<0.038$ ).

\section{Reclassification from low-flow to normal-flow AS} Imaging data fusion method reclassified $24.1 \%$ of patients from a low-flow to a normal-flow AS group. Reclassification from severe to non-severe AS by IDFM was accompanied by the reclassification from a low-flow to a normal-flow group in three out of four patients. In one case, SVFi remained in the low-flow range. Six (11.1\%) patients in the NFHG group, one $(1.9 \%)$ patient in the NFLG group, three (5.6\%) patients in the LFLG group, and one in the LFHG group were reclas- 




Figure 2. Linear correlations and Bland-Altman analyses of calculations of indexed stroke volume (SVi) and indexed aortic valve area (AVAi) on multislice computed tomography (MSCT) and two-dimensional transthoracic echocardiography (2D-TTE). Fusion MSCT SVi (MSCT SVFi circ) and AVAi (MSCT AVAFi circ) were calculated with the left ventricular outflow tract area evaluated from a maximal and minimal diameter with the $\pi r^{2}$ formula, where $r$ was $1 / 2$ of the mean diameter. Pearson correlations showed high agreement between MSCT (MSCT SVFi circ) and 2D-TTE (2D-TTE SVi) calculations of the indexed stroke volume (A) and between MSCT (MSCT AVAFi circ) and 2D-TTE (2D-TTE AVAi) calculations of the indexed aortic valve area (B). The Bland-Altman plots demonstrate that SVi and AVAi are underestimated by 2D-TTE compared with MSCT (C, D). Echocardiography underestimated SVi and AVAi on average by $34 \%$ compared with MSCT

sified to non-severe AS. None of the analysed variables was an independent predictor of reclassification from low-flow to normal-flow AS group.

\section{DISCUSSION}

The correct assessment of distal LVOT diameters and area in the MSCT enabled the recalculation of SV and AVA in IDFM. Similarly to others, we demonstrated that the 2D-TTE LVOT diameter $(20.3 \pm 2.3 \mathrm{~mm})$ actually represented the minimal MSCT LVOT diameter $(20.2 \pm 2.8 \mathrm{~mm})$, yielding an underestimation of the 2D-TTE LVOT area, SV, and finally AVA [6-8]. The TAVI population was chosen deliberately. All patients underwent 2D-TTE, 2D-TEE, 3D-TEE, and MSCT, which enabled the comparison of all four imaging methods and related our findings to the observations of Kamperidis et al. [8] on a TAVI population. We were aware that reclassification of AS severity makes sense especially in low-gradient groups, but we wanted to test it in all patients to potentially establish a new cut-off point for the diagnosis. We preferred AVAi, as did Kamperidis et al. [8], because it is a more precise parameter than straight AVA and can be used in patients with different body weight and height as well as for the purpose of comparison between authors.

In the current study, recalculation of AVAi with IDFM resulted in a potential change of the diagnosis from severe to non-severe AS in $20.4 \%$ of patients. In the study by Kamperidis 


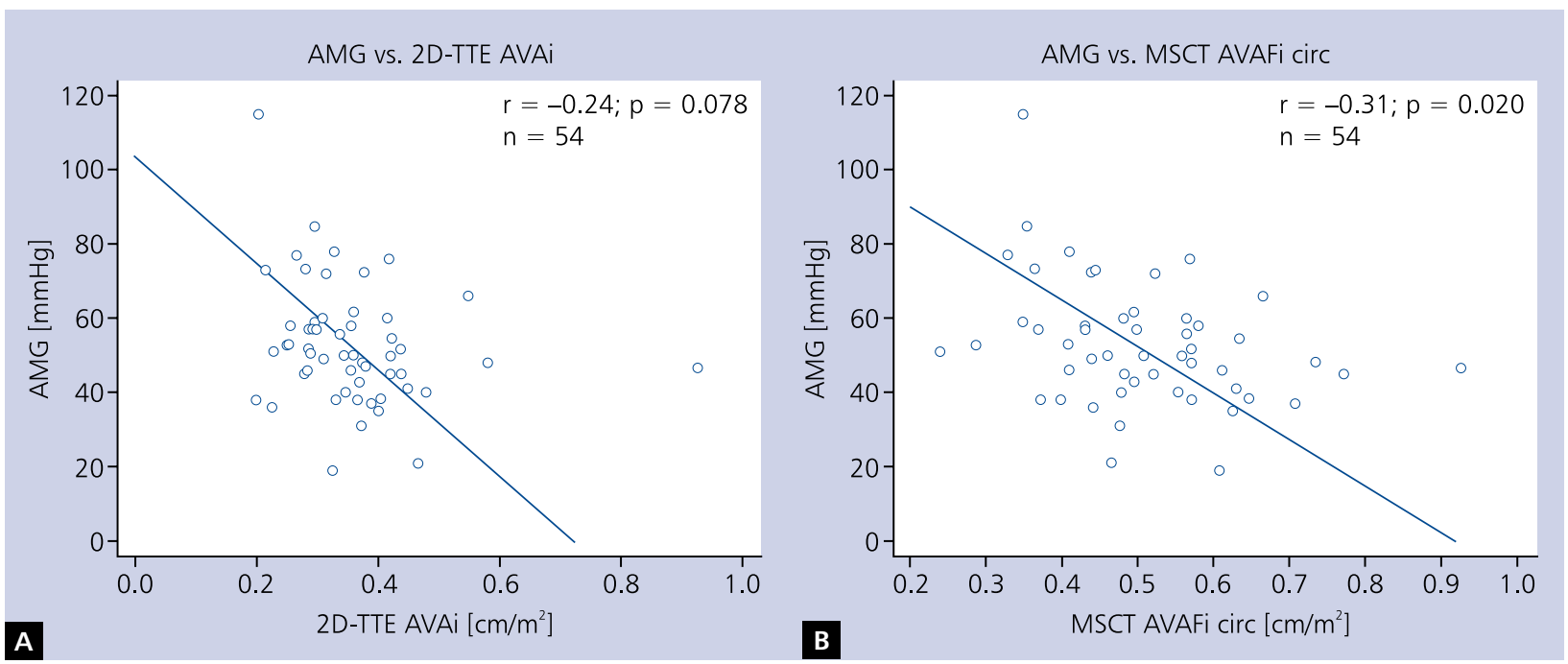

Figure 3. Non-linear correlations between aortic mean gradient (AMG) and indexed aortic valve area (AVAi) for two-dimensional transthoracic echocardiography (2D-TTE) and multislice computed tomography (MSCT). AVAi was calculated by Doppler echocardiography (2D-TTE AVAi) (A) or by MSCT with the left ventricular outflow tract area evaluated from a maximal and minimal diameter with the $\pi r^{2}$ formula, where $r$ was $1 / 2$ of the mean diameter (MSCT AVAFi circ) (B). The correlation between AMG and AVAi was better for MSCT AVAFi circ than 2D-TTE AVAi and was statistically significant

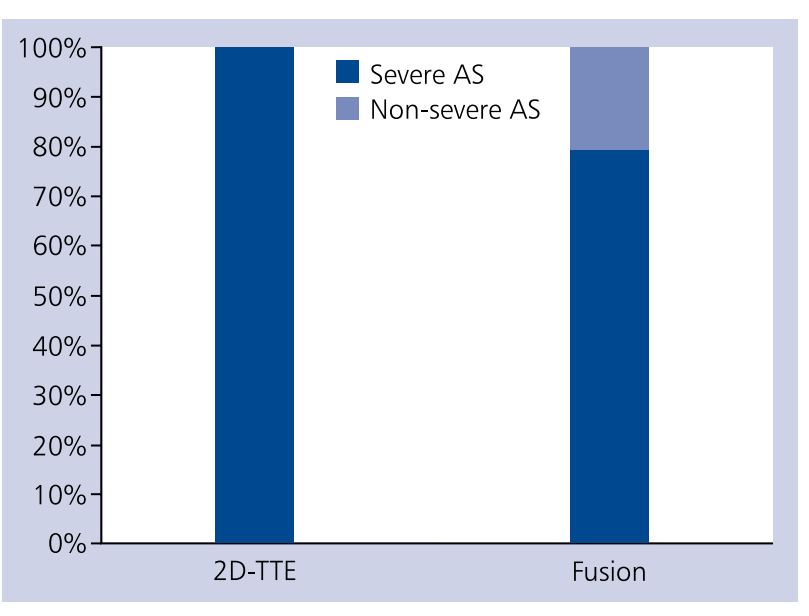

Figure 4. Aortic stenosis (AS) severity reclassification with imaging data fusion method (IDFM) combining multislice computed tomography of the left ventricular outflow tract area and twodimensional transthoracic echocardiography (2D-TTE) Doppler parameters. Calculated from a minimal and maximal diameter with the $\pi r^{2}$ formula, where $r$ was $1 / 2$ of the mean diameter. IDFM reclassified $20.4 \%$ of patients into non-severe AS group

et al. [8], $16.2 \%$ of TAVI patients were reclassified by IDFM (with the same threshold of AVAFi $\geq 0.6 \mathrm{~cm}^{2} / \mathrm{m}^{2}$ ). The difference might be explained by the fact that Kamperidis et al. [8] derived their calculations from the planimetered LVOT area. Adopting the same method would have resulted in a nearly identical reclassification rate in our study (16\%).

Overall, low-gradient AS was present in approximately one in every five patients. This is comparable to other studies
$[14,15]$. In the current study, IDFM reclassified $25 \%$ of patients from severe to non-severe AS in the NFLG subgroup and as much as $33 \%$ in the LFLG subgroup when using MSCT LVOT planimetered area. These results differ from those reported in the study by Kamperidis et al. [8] in which these rates were $52 \%$ and $11.9 \%$, respectively. The reasons for these differences are unclear, but may be partially explained by the differences in the body surface areas and 2D-TTE-calculated and MSCT-planimetered LVOT areas in the studied populations. Although in our study the number of patients who were reclassified to AVAFi $\geq 0.6 \mathrm{~cm}^{2} / \mathrm{m}^{2}$ was highest in the LFLG subgroup (even $50 \%$ when LVOTarea ${ }_{\text {MSCTcirc }}$ was used), IDFM reclassified also as many as $17.6 \%$ of the NFHG and $10 \%$ of the LFHG patients. When it was calculated with MSCT-planimetered LVOT area the results were $14.7 \%$ and $10 \%$, respectively. Kamperidis et al. [8] reported much lower rates for the NFHG and LFHG groups: $2.8 \%$ and $0 \%$, respectively. This may be explained by the fact that 2D-TTE and MSCT LVOT areas in the NFHG and LFHG groups were apparently larger in the current study than in the series by Kamperidis et al. [8], and these differences translated to a reclassification of AVAF and AVAFi. In comparison, in our study 2D-TEE enabled the reclassification of only $3.7 \%$ of TAVI patients (with no subjects from the low-gradient subgroups) and 3D-TEE reclassified only $11.1 \%$ of them (with only one [1.9\%] patient from the low-gradient subgroup). An important conclusion from the current study is that reclassification with IDFM may affect not only patients with low-gradient AS, but also a considerable number of patients with significantly elevated transaortic gradients.

It should be pointed out that the emergence of IDFM calls for diagnostic caution in establishing AS severity $[7,16]$. 
Table 4. Predictors of reclassification to moderate aortic stenosis in imaging data fusion method for two-dimensional transthoracic echocardiography and multislice computed tomography (MSCT). Univariable model

\begin{tabular}{|c|c|c|c|}
\hline & OR & $95 \% \mathrm{Cl}$ & $p$ \\
\hline Age [years] & 0.85 & $0.73-0.98$ & $<0.029$ \\
\hline Male sex [\%] & 5.52 & $1.27-24.08$ & $<0.023$ \\
\hline \multicolumn{4}{|l|}{ CAD: } \\
\hline with previous MI [\%] & 5.78 & $1.40-23.84$ & $<0.015$ \\
\hline with previous CABG [\%] & 7.40 & $1.71-32.09$ & $<0.008$ \\
\hline Diabetes mellitus [\%] & 13.09 & $2.44-70.12$ & $<0.003$ \\
\hline $\mathrm{VTI}_{\text {LVOT }}[\mathrm{cm}]$ & 1.14 & $1.00-1.3$ & $<0.047$ \\
\hline $\mathrm{AMG}[\mathrm{mmHg}]$ & 0.94 & $0.88-1.0$ & $<0.036$ \\
\hline LVOT $_{\text {MSCTmin }}[\mathrm{mm}]$ & 1.45 & $1.06-1.98$ & $<0.019$ \\
\hline LVOT $_{\text {MScTmean }}[\mathrm{mm}]$ & 1.36 & $1.03-1.80$ & $<0.032$ \\
\hline LVOTarea $_{\text {MSCTirc }}\left[\mathrm{mm}^{2}\right]$ & 1.01 & $1.00-1.02$ & $<0.028$ \\
\hline LVOTarea $_{\text {MScTplanimetry }}\left[\mathrm{mm}^{2}\right]$ & 1.01 & $1.00-1.01$ & $<0.047$ \\
\hline AAarea $_{\text {MscTplanimetry }}\left[\mathrm{mm}^{2}\right]$ & 1.01 & $1.00-1.02$ & $<0.021$ \\
\hline
\end{tabular}

AAarea $_{\text {MSCTplanimetry }}$ - aortic annulus area planimetered in MSCT; $\mathrm{Cl}$ - confidence interval; OR — odds ratio; other abbreviations - see Tables 1 and 2

Jander et al. [17] observed that fusion effective AVA is larger than the corresponding anatomic AVA and adjustment of partition values may be warranted when using IDFM. Clavel et al. [7] proposed a new 1.2- $\mathrm{cm}^{2}$ AVA cut-off point for assessing AS severity in MSCT. Many authors [5, 6] observed that non-indexed AVA calculated with IDFM was greater than AVA calculated with 2D-TTE, by about 0.1 to $0.2 \mathrm{~cm}^{2}$. In our study this difference was $0.15 \mathrm{~cm}^{2}$ (seven patients had $A V A F \geq 1.2 \mathrm{~cm}^{2}$ out of 11 patients with AVAFi $\geq 0.6 \mathrm{~cm}^{2} / \mathrm{m}^{2}$ ). Clavel et al. [7] noticed significant correlations between AMG and non-indexed AVA in a wide range of AVAs (from below 0.5 to over $3.0 \mathrm{~cm}^{2}$ ), both for 2D-TTE measurements and for IDFM, without significant differences in the correlation strength between both methods. In the current study, we observed a significant correlation between AMG and AVAFi, but this was not true for 2D-TTE.

In the current study, we determined the predictors of AS severity reclassification from severe to moderate. In a univariable model the reclassification was independently associated with younger age, male sex, CAD with MI, CABG, DM, and a lower $\mathrm{VTI}_{\mathrm{AV}}$ and $\mathrm{AMG}$ values as well as higher $\mathrm{VTI}_{\mathrm{LVOT}^{\prime}}$

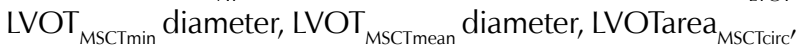
LVOTarea $_{\text {MSCTplanimetry' }^{\prime} \text { and AAarea }}$ MSCTplanimetry values. In a multivariable model AS reclassification was independently associated with age, DM, $\mathrm{VTI}_{\mathrm{LVOT}}, \mathrm{AMG}$, and MSCT LVOT minimal diameter. This calls for caution when establishing the diagnosis of severe AS based on 2D-TTE, especially in younger patients and diabetics, as well as in those with a higher $\mathrm{VTI}_{\mathrm{LVOT}}$ and lower AMG and a relatively larger LVOT. While it is self-explanatory that a larger LVOT and higher $\mathrm{VTI}_{\text {LVOT }}$ values are associated with a greater risk of reclassification, this is not the case for age and DM. The question as to whether the latter is related to greater peripheral resistance in diabetic patients remains to be established [18].

The imaging data fusion method reclassified nearly a quarter of the studied patients from the low-flow to the normal-flow AS group. As in the study by Kamperidis at al. [8], not every SVi increase above the $35-\mathrm{mL} / \mathrm{m}^{2}$ threshold was associated with AVAi reclassification. No specific predictors of this reclassification were found.

Our results represent a single-centre experience. The small sample size limited our ability to build complex multivariable models and subgroup analyses. It seems that the validation of IDFM would come from big TAVI registries; with a large number of patients the AVAFi cut-off point could be established. AVAFi and the predictors of reclassification could be matched with short- and long-term survival.

In summary, IDFM led to the reclassification of AS severity from severe to potentially non-severe in approximately one in every five patients. Clinical and anatomic predictors of such reclassification were established. Reclassification of AVA values by IDFM calls for caution but should be considered especially in cases of low-gradient AS, i.e. "difficult track" AS, according to the recent guidelines [11], particularly in patients in whom the probability of AS or flow status reclassification is high.

\section{Acknowledgements}

The authors thank Robin Krauze and Professor Tadeusz Krauze as well as Mathieu Spencer for their invaluable remarks and language help.

Conflict of interest: none declared 


\section{References}

1. Généreux P, Head SJ, Wood DA, et al. Transcatheter aortic valve implantation 10-year anniversary: review of current evidence and clinical implications. Eur Heart J. 2012; 33(19): 2388-2398, doi: 10.1093/eurheartj/ehs220, indexed in Pubmed: 22851654.

2. Indraratna P, Tian DH, Yan TD, et al. Transcatheter aortic valve implantation versus surgical aortic valve replacement: A meta-analysis of randomized controlled trials. Int J Cardiol. 2016; 224: 382-387, doi: 10.1016/j.ijcard.2016.09.018, indexed in Pubmed: 27673695.

3. Jilaihawi H, Doctor N, Kashif M, et al. Aortic annular sizing for transcatheter aortic valve replacement using cross-sectional 3-dimensional transesophageal echocardiography. J Am Coll Cardiol. 2013; 61(9): 908-916, doi: 10.1016/j.jacc.2012.11.055, indexed in Pubmed: 23449425.

4. Achenbach S, Delgado V, Hausleiter J, et al. SCCT expert consensus document on computed tomography imaging before transcatheter aortic valve implantation (TAVI)/transcatheter aortic valve replacement (TAVR). J Cardiovasc Comput Tomogr. 2012; 6(6): 366-380, doi: 10.1016/j.jcct.2012.11.002, indexed in Pubmed: 23217460.

5. Saitoh T, Shiota M, Izumo M, et al. Comparison of left ventricular outflow geometry and aortic valve area in patients with aortic stenosis by 2-dimensional versus 3-dimensional echocardiography. Am J Cardiol. 2012; 109(11): 1626-1631, doi: 10.1016/j. amjcard.2012.01.391, indexed in Pubmed: 22440128.

6. Gaspar T, Adawi S, Sachner R, et al. Three-dimensional imaging of the left ventricular outflow tract: impact on aortic valve area estimation by the continuity equation. J Am Soc Echocardiogr. 2012; 25(7): 749-757, doi: 10.1016/j.echo.2012.05.001, indexed in Pubmed: 22613774.

7. Clavel MA, Malouf J, Messika-Zeitoun D, et al. Aortic valve area calculation in aortic stenosis by CT and Doppler echocardiography. JACC Cardiovasc Imaging. 2015; 8(3): 248-257, doi: 10.1016/j.jcmg.2015.01.009, indexed in Pubmed: 25772832.

8. Kamperidis V, van Rosendael PJ, Katsanos S, et al. Low gradient severe aortic stenosis with preserved ejection fraction: reclassification of severity by fusion of Doppler and computed tomographic data. Eur Heart J. 2015; 36(31): 2087-2096, doi: 10.1093/eurheartj/ehv188, indexed in Pubmed: 26033985.

9. Tzikas A, Schultz CJ, Piazza N, et al. Assessment of the aortic annulus by multislice computed tomography, contrast aortography, and trans-thoracic echocardiography in patients referred for transcatheter aortic valve implantation. Catheter Cardiovasc Interv. 2011; 77(6): 868-875, doi: 10.1002/ccd.22761, indexed in Pubmed: 20824762.
10. Hahn R. Use of imaging for procedural guidance during transcatheter aortic valve replacement. Curr Opin Cardiol. 2013; 28(5): 512-517, doi: 10.1097/hco.0b013e3283632b5e.

11. Baumgartner H, Hung J, Bermejo J, et al. Recommendations on the echocardiographic assessment of aortic valve stenosis: a focused update from the European Association of Cardiovascular Imaging and the American Society of Echocardiography. Eur Heart J Cardiovasc Imaging. 2017; 18(3): 254-275, doi: 10.1093/ehjci/jew335, indexed in Pubmed: 28363204.

12. Evangelista A, Flachskampf F, Lancellotti P, et al. European Association of Echocardiography recommendations for standardization of performance, digital storage and reporting of echocardiographic studies. Eur J Echocardiogr. 2008; 9(4): 438-448, doi: 10.1093/ejechocard/jen174, indexed in Pubmed: 18579482.

13. Lang R, Badano L, Mor-Avi V, et al. Recommendations for Cardiac Chamber Quantification by Echocardiography in Adults: An Update from the American Society of Echocardiography and the European Association of Cardiovascular Imaging. Eur Heart J Cardiovasc Imaging. 2015; 16(3): 233-271, doi: 10.1093/ehjci/jev014.

14. Clavel MA, Dumesnil JG, Capoulade R, et al. Outcome of patients with aortic stenosis, small valve area, and low-flow, low-gradient despite preserved left ventricular ejection fraction. J Am Coll Cardiol. 2012; 60(14): 1259-1267, doi: 10.1016/j.jacc.2011.12.054, indexed in Pubmed: 22657269.

15. Mohty D, Magne J, Deltreuil M, et al. Outcome and impact of surgery in paradoxical low-flow, low-gradient severe aortic stenosis and preserved left ventricular ejection fraction: a cardiac catheterization study. Circulation. 2013; 128(11 Suppl 1): S235-S242, doi: 10.1161/CIRCULATIONAHA.112.000031, indexed in Pubmed: 24030412.

16. Flachskampf FA. Stenotic aortic valve area: should it be calculated from CT instead of echocardiographic data? JACC Cardiovasc Imaging. 2015; 8(3): 258-260, doi: 10.1016/j.jcmg.2014.12.012, indexed in Pubmed: 25772833.

17. Jander N, Wienecke S, Dorfs S, et al. Anatomic estimation of aortic stenosis severity vs "fusion" of data from computed tomography and Doppler echocardiography. Echocardiography. 2018; 35(6): 777-784, doi: 10.1111/echo.13855, indexed in Pubmed: 29522643.

18. Suzuki E, Yoshimura T, Omura Y, et al. Higher arterial stiffness, greater peripheral vascular resistance and lower blood flow in lower-leg arteries are associated with long-term hyperglycaemia in type 2 diabetic patients with normal ankle-brachial index. Diabetes Metab Res Rev. 2009; 25(4): 363-369, doi: 10.1002/dmrr.955, indexed in Pubmed: 19334018

Cite this article as: Stokłosa P, Michałowska I, Duchnowski P, et al. Predictors of aortic stenosis severity reclassification using an imaging data fusion method in patients referred for transcatheter aortic valve implantation. Kardiol Pol. 2018; 76(12): 1725-1732, doi: 10.5603/KP.a2018.0195.

\section{WHAT IS NEW?}

The use of the imaging data fusion method (combining two-dimensional transthoracic echocardiography and multislice computed tomography [MSCT] parameters) led to the reclassification of aortic stenosis severity from severe to potentially non-severe in approximately one in every five patients - including patients with high gradients. Clinical (younger age and diabetes mellitus) and anatomic predictors (minimal left ventricular outflow tract diameter on MSCT) of such reclassification were established. Reclassification of aortic valve area values using the imaging data fusion method calls for caution but should be considered especially in cases of low-gradient aortic stenosis, i.e. "difficult track" aortic stenosis, particularly in patients with a high probability of aortic stenosis severity or flow status reclassification. 